Original Article

\title{
Comparing Mean Post-Operative Back Pain Score between Hemilaminectomy and Conventional Laminectomy in Patients of Lumbar Stenosis
}

Taimoor Anwar ${ }^{1}$, Muhammad Abdur Rehman ${ }^{1}$, Urwa Tanveer Ahmad ${ }^{1}$, Ammar Anwer ${ }^{1}$ Anum Wahab ${ }^{1}$, Qura Tul Ain Tariq ${ }^{3}$, Zunera Tanveer ${ }^{4}$, Mohammad Zahid Mustafa ${ }^{5}$

${ }^{1}$ Department of Neurosurgery, ${ }^{2}$ Allied Burn and Reconstructive Surgery Center, Allied Hospital, Faisalabad, ${ }^{3}$ Government General Hospital, Ghulam Muhammadabad, Faisalabad, ${ }^{4}$ Department of Physiology, Bolan University of Medical and Health Sciences, ${ }^{5}$ Center for Advanced Studies in Vaccinology and Biotechnology (CASVAB), University of Baluchistan, Quetta - Pakistan

\section{ABSTRACT}

Objective: To observe the comparative mean post-operative back-pain score between hemilaminectomy and conventional laminectomy in patients of lumbar stenosis.

Material and Methods: The randomized controlled study was carried out in Neurosurgery Unit, Allied Hospital, Faisalabad, Pakistan. A total of 60 patients were distributed equally in two groups. Group A underwent hemilaminectomy while group B underwent conventional laminectomy. A linear median fascial incision was made on the side with more pain or symptoms. Only in the hemilaminectomy group, ipsilateral decompression was performed. It involves partial resection of adjacent parts of the hemi laminae of the superior and inferior vertebrae using operative loupes or neurosurgical microscope. Mean \pm SD was calculated for quantitative data including back pain score.

Results: Mean age was $46.2 \pm 6.94$ years in hemi group and $46.3 \pm 6.74$ years in the conventional group. We observed that in hemi group, the back pain score was $2.23 \pm 0.73$ and it was $2.7 \pm 0.65$ in the conventional group ( $p$-value was 0.011). Significant differences ( $p$ value $<0.050)$ existed in these age ranges with respect to the back pain score in both surgery groups. A significant difference ( $p$ value $<0.0001$ ) observed only in male patients between two surgery groups for the back pain scores.

Conclusion: Mean post-operative back pain score is significantly reduced in hemilaminectomy cases when compared with conventional laminectomy in patients of lumbar stenosis.

Keywords: Lumbar spinal stenosis, hemilaminectomy, conventional laminectomy, postoperative back pain.

Corresponding Author: Muhammad Abdur Rehman

Department of Neurosurgery

Allied Hospital, Faisalabad - Pakistan

Email: doc_maan87@yahoo.com
Date of Submission: 26-04-2021

Date of Revision: 25-05-2021

Date of Online Publishing: 13-06-2021

Date of Print: 30-06-2021

DOI: $10.36552 / p j n s . v 25 i 2.555$ 


\section{INTRODUCTION}

Conventional laminectomy is the commonest surgical approach for spinal canal decompression. A major proportion of patients with lumbar stenosis get surgical decompression. Decompression surgery for this condition has promising results in more than eighty percent of patients in terms of back pain reduction. Lumbar stenosis is a pathological condition of the spine resulting from spondylosis. ${ }^{5}$ It can involve the whole of the spine but mostly the cervical spine and the lumbar part of the spine are involved. As these parts of the spine a relatively more mobile and bear weight of the body, these are commonly complicated by degenerative changes due to arthritis. $^{11}$

Degeneration with lumbar stenosis can occur due to disturbance of any bony part, disc, and capsules around the joints or ligaments around the joints holding the spine. ${ }^{4}$ Spinal stenosis of the lumbar part is more frequent in obese and old patients. Multiple issues disturbing the normal anatomy of the spine including the extra-growths around the facet joints, enlargement of the ligamentum flavum, destruction for the intervertebral discs and abnormal bony projections make the canal in the spine narrower than normal, ending up in the compression of the cord and nerve roots. ${ }^{12}$

Incidence of lumbar stenosis ranges from $4 \%$ to $11 \% .{ }^{6}$ A large number of patients are treated through surgical decompression with satisfactory results. More than eighty percent of the patients with this condition, improve successfully through decompression surgery. ${ }^{14}$ But it should be done after the failure of the medical and physiotherapy conservative management. ${ }^{2}$ Current prevailing approaches include laminectomy with or without fusion, laminotomy with medial facetectomy, laminoplasty, minimally invasive bilateral decompression, unilateral laminectomy with bilateral decompression, and placement of an interspinous device. ${ }^{8}$ Conventional laminectomy is mostly done in surgical management for decompression of the cord in the spinal canal but at the cost of spinal, paraspinal, interspinous, and supraspinous structures. ${ }^{2}$

Young et al. (1988) described the unilateral approach for the first time ${ }^{13}$ and later this technique was modified by McCulloch. This microscopic technique is characterized by ipsilateral and contralateral micro-decompression involving the posterior structures in or next to the midline. The unilateral approach preserves facet joints and the neural arches, thus maintaining post-operative stability and does not cause scarring. ${ }^{9}$

In a study, post-operative back pain visual analog score after 6 months hemilaminectomy was noted to be $3.5 \pm 0.8$ and with conventional laminectomy, it was $3.6 \pm 0.10$. In another study, post-operative back pain visual analog score after 6 months with unilateral the approach was observed as $1.73 \pm 0.61$ and with conventional laminectomy it was $3.33 \pm 0.59 .^{12}$ Literature shows controversial treatment options for lumbar stenosis with regards to postoperative back pain. So, this study was conducted to evaluate a better treatment option with minimal post-operative problems for patients with spinal stenosis. This study aimed to observe the comparative mean post-operative back-pain score between hemilaminectomy and conventional laminectomy in patients with lumbar stenosis.

\section{MATERIAL \& METHODS}

\section{Study Design \& Setting}

This randomized controlled study was carried out in Neurosurgery Unit, Allied hospital, Faisalabad, Pakistan. The study was conducted after proper approval from the hospital ethical committee for research/clinical trials. The duration of the study was 6 months after the completion of the approval process. 


\section{Groups of Patients}

Patients were enrolled after written informed consent along with an explanation of the whole procedure of the study. Patients were randomly categorized into two groups with the help of computer numbers.

Group A: Patients underwent hemilaminectomy.

Group B: Patients underwent conventional laminectomy.

\section{Inclusion Criteria}

All those patients with MRI or computed tomography (CT) showed any type of disc herniation.

The trail was conducted on the patients ( $n=$ 60) of both genders, age between 20 - 60 years with lumbar stenosis having ASA grade I. Lumbar stenosis was defined as the patients with symptoms of neurogenic claudication, radiological/neuroimaging evidence of lumbar spinal stenosis (LSS) involving the central canal and/or foraminal stenosis on MRI.

\section{Exclusion Criteria}

Those having lumbar isthmic spondylolysis, lumbar spondylolisthesis of grade above I and those patients with previous lumbar surgery, along with those cases having psychiatric disorders, alcohol abuse, on drugs, and patients with bilateral radiculopathy were not included in the study.

\section{Surgical Procedure}

Following general anesthesia, all the patients were placed in the prone position, level of stenosis was confirmed and a midline incision was given.

\section{Group A}

In the hemilaminectomy group, ipsilateral decompression was performed with the help of microscope or operative loupes. Partial resection was done at the inferior aspect of the cranial hemi lamina along with the superior part of the caudal hemi lamina. The base of the spinous process was undercut and the base of contralateral hemilamina was also cut. Later on, flavectomy was done on both sides, and the lateral recess and neural foramina were decompressed contralaterally. The procedures were performed by senior neurosurgeons.

\section{Group B}

Conventional laminectomy and decompression was performed.

Post-operative back pain scoring was done after six months of the procedure. Follow-up was ensured by taking the patient's contact number.

\section{Post-Surgical Evaluations}

After the operation/procedure the back the pain was assessed using a scoring system of visual analog scale (VAS) Score from 0 (patients with no pain were labeled as 0 zero score) to 10 (patients with worst possible pain anyone can have) after six months of treatment.

\section{Data Analysis}

Data were transferred on excel sheets and analyzed using SPSS V-25. Mean \pm SD was calculated for quantitative data including back pain score. Percentages were calculated for qualitative variables including ASA status. The stratified data with regard to the age groups and gender were assessed through Chi-square $\left(x^{2}\right)$ test to find any relative difference of significance. The comparison of post-operative back pain scores of both groups was done using an independent Sample t-test. Effect modifiers like age, gender, and ASA status were controlled by stratification. Post-stratification independent sample $\mathrm{t}$-test was applied. A $\mathrm{p}$-value $\leq 0.05$ was taken as significant. 


\section{RESULTS}

All of the 60 subjects (Group $A ; n=30$, Group B; $n=30$ ), fulfilling the defined study criteria were evaluated for mean postoperative back pain score between hemilaminectomy and conventional laminectomy in patients of lumbar stenosis.

\section{Age Distribution}

In all study subjects' distribution of the age showed that $30 \%(n=9)$ in the hemi group and $33.3 \%$ $(n=10)$ in the conventional group were between $20-40$ years. While, $70 \%(n=21)$ in the hemi group and $66.67 \%$ $(n=20)$ in the conventional group were $40-60$ years of age. Mean \pm SD for age was $46.2 \pm 6.94$ years in the hemi group and $46.3 \pm 6.74$ years in the conventional group (Table 1). An insignificant difference $(p<0.050)$ existed between age-group distribution for both groups.

\section{Gender Distribution}

$50 \%(n=15)$ in hemi group and 53.3\% $(n=16)$ in conventional group were male, whereas $50 \%(n=$ $15)$ in hemi group and $46.67 \%(n=14)$ in conventional group were females (Table 2). An insignificant difference $(p<0.050)$ existed between gender distribution for both groups.

\section{Comparison: Mean Post-Operative Back Pain Scores in Surgery Groups}

A comparison of mean post-operative back pain score between hemilaminectomy and conventional laminectomy in patients of lumbar stenosis was done. We observed that in hemi group, the back pain score was $2.23 \pm 0.73$ and it was $2.7 \pm 0.65$ in conventional group ( $p$-value was 0.011) (Table 3).

Table 4 shows the stratification for comparison of mean post-operative back pain score between hemilaminectomy and conventional laminectomy in patients of lumbar stenosis with regards to two age ranges: $20-40$ years and 41-60 years. Significant differences ( $p$ value $<0.050$ ) existed in these age ranges with respect to the back pain score in both surgery groups. 
Table 5 shows the stratification for comparison of mean post-operative back pain score between hemilaminectomy and conventional laminectomy in patients of lumbar stenosis with regards to gender. A significant difference ( $p$ value $<0.0001$ ) observed only in male patients between two surgery groups for the back pain scores.

Table 4: Comparison of mean post-operative back pain score between hemilaminectomy and conventional laminectomy with regards to age $(n=60)$.

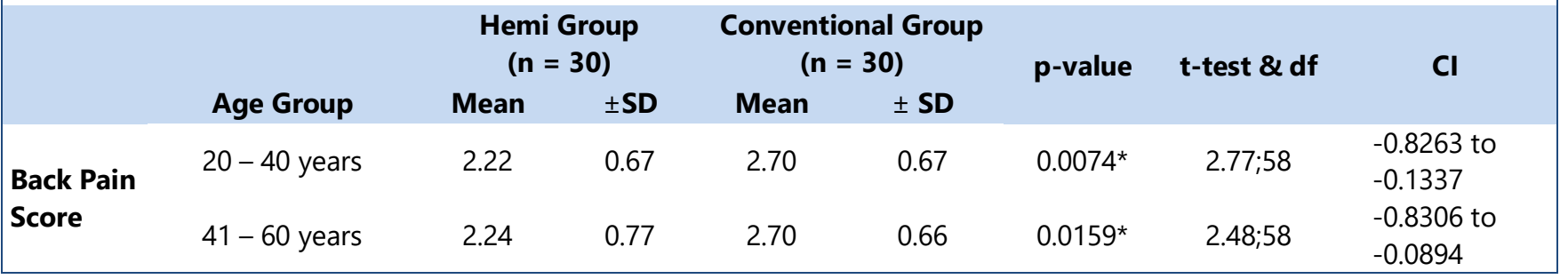

*significant

Table 5: Comparison of mean post-operative back pain score between hemilaminectomy and conventional laminectomy with regards to gender $(n=60)$.

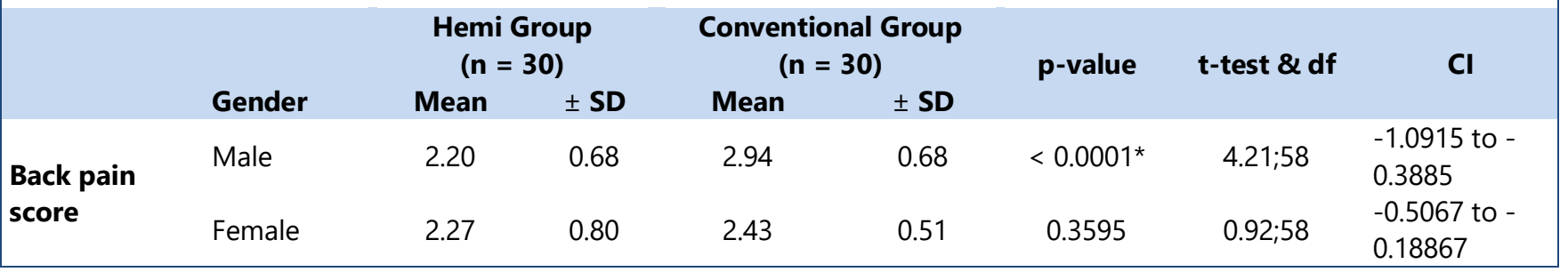

*significant

\section{DISCUSSION}

Lumbar spinal stenosis (LSS) is commonly seen in the elderly. Facet joint degeneration, thickening of ligamentum flavum, formations of osteophytes, destruction of the disc, narrowing of the spinal canal, and compression of roots lead to pain and other symptoms. Initially managed with a conservative approach and later surgical decompression through conventional laminectomy is offered. The conventional (bilateral) approach in this procedure leads to the loss of multiple structures including spinal muscles, bilateral lamina, and facet joints. This microscopic technique is characterized by ipsilateral and contralateral micro-decompression performed in the posterior midline structures. The advantage of the unilateral approach includes preservation of neural arches and facet joints and maintains post-operative stability of the spine. There is no scar formation. Literature shows controversy between the conventional laminectomy and hemilaminectomy for treatment of lumbar stenosis in terms of post-operative back pain. ${ }^{9}$ So, this study was planned to evaluate a better treatment modality for patients with lumbar stenosis with less post-operative back pain which can be offered to all the patients.

The results of this study were compared with the observations of $\mathrm{Hu}$ et al, where the postoperative back pain visual analog score after 6 
months' hemilaminectomy was $3.5 \pm 0.8$ and with conventional laminectomy it was $3.6 \pm 0,{ }^{10}$ while we observed the post-operative back pain visual analog score after 6 months with hemilaminectomy $2.23 \pm 0.73$ and with conventional laminectomy it was $2.7 \pm 0.65$. In another study, post-operative back pain visual analog score after 6 months with unilateral approach was observed as $1.73 \pm 0.61$ and with conventional laminectomy it was $3.33 \pm 0.59 .{ }^{4}$ Furthermore, the mean postoperative back pain score, in patients receiving conventional laminectomy, was $2.90 \pm 0.97$, while it was $2.67 \pm$ 0.50 in hemi $^{10}$ and this is compatible with the mean post-operative back pain score of our findings. Though in our study, a higher difference was not recorded, it was statistically significant.

The satisfactory results in all study subjects were reported in another study, where the efficacy of the mini-invasive spinal technique for spinal stenosis of the lumbar part through unilateral approach was compared with the conventional surgical approach. ${ }^{9}$ Additionally, the unilateral approach with hemilaminectomy showed a shorter mean hospital stay and better recovery time in more than eighty percent of patients. Five of these cases had some major complications, two had un-intended dural rent, while two others had wound dehiscence and the fifth one showed the failure of surgical treatment and complicated symptoms. This approach has an additional advantage of vertebral stability. It involves minimum muscular clearance and early chances of post-operative return of the patient to routine activity, by preserving spinal muscles, ligaments, and the spinous process. This approach leads because of its shorter hospital stay, reduced postoperative back pain, and better patient satisfaction. ${ }^{9}$

Costa et al, also concluded that on comparison of the clinical outcomes of unilateral laminotomy-for-bilateral decompression observed significant improvement in patients with lumbar stenosis. ${ }^{1}$ Besides, the preservation of contralateral paraspinal muscles and pars interarticularis reduces the potential complications like postoperative infection and cicatrization by decreased dead space as well as enhanced stability and reduce post-operative back pain.,10 Considering the above facts regarding postoperative we found that there is some difference between hemilaminectomy and conventional laminectomy in patients of lumbar stenosis in terms of mean postoperative back pain score. However, being the limitation of our study, we did not compare the success rate of hemilaminectomy with conventional laminectomy, which should be addressed in coming trials.

\section{CONCLUSION}

We concluded that mean post-operative back pain score is significantly reduced in hemilaminectomy cases when compared with conventional laminectomy in patients of lumbar stenosis.

\section{Conflict of Interest}

Authors have no conflict of interest.

\section{REFERENCES}

1. Costa F, Sassi M, Cardia A, Ortolina A, De Santis A, Luccarell G, Fornari M: Degenerative lumbar spinal stenosis: analysis of results in a series of 374 patients treated with unilateral laminotomy for bilateral microdecompression. Journal of Neurosurgery: Spine, 2007; 7 (6): 579-86.

2. Haddadi K, Ganjeh Qazvini HR. Outcome after surgery of lumbar spinal stenosis: a randomized comparison of bilateral laminotomy, trumpet laminectomy, and conventional laminectomy. Frontiers in Surgery, 2016; 8 (3): 19.

3. Hu W, Zhao J, Gong C, Zou M, Yuan JH, Liu XY: A comparation of efficacy between unilateral laminectomy approach bilateral decompression and traditional total laminectomy decompression in the treatment of lumbar spinal stenosis. 
Zhonghuayixuezazhi, 2016; 96 (21): 1673-6.

4. Komp M, Hahn P, Oezdemir S, Giannakopoulos A, Heikenfeld $R$, Kasch $R$, Merk $H$, Godolias $G$, Ruetten S: Bilateral spinal decompression of lumbar central stenosis with the full-endoscopic interlaminar versus microsurgical laminotomy technique: a prospective, randomized, controlled study. Pain Physician, 2015; 8 (1): 61-70.

5. Moisi M, Fisahn C, Tkachenko L, Tubbs RS, Ginat D, Grunert P, Jeyamohan S, Reintjes S, Ajayi O, Page J, Oskouian RJ: Unilateral laminotomy with bilateral spinal canal decompression for lumbar stenosis: a technical note. Cureus, 2015; 8 (5): e623.

6. Munting $E$, Röder C, Sobottke R, Dietrich $D$, Aghayev E: Patient outcomes after laminotomy, hemilaminectomy, laminectomy and laminectomy with instrumented fusion for spinal canal stenosis: a propensity score-based study from the Spine Tango registry. European Spine Journal, 2015; 24 (2): 358-68.

7. Rahman $M$, Summers LE, Richter $B$, Mimran RI, Jacob RP: Comparison of techniques for decompressive lumbar laminectomy: the minimally invasive versus the "classic" open approach. minMinimally Invasive Neurosurgery, 2008; 51 (02): 100-5.

8. SHin $M H$, KiM JS, Ryu KS, HuR JW: Bilateral Decompression via Microscopic Tubular Crossing Laminotomy (MTCL) for Lumbar Spinal Stenosis: Technique and Early Surgical Result. Neurologia
Medico-Chirurgica, 2015; 55 (7): 570-7.

9. Usman M, Ali M, Khanzada K, Ishaq M, Naeem-ulHaq AR, Ali M: Unilateral approach for bilateral decompression of lumbar spinal stenosis: a minimal invasive surgery. J Coll Physicians Surg Pak. 2013; 23 (12): 852-856.

10. Weiner BK, Walker M, Brower RS, McCulloch JA: Microdecompression for lumbar spinal canal stenosis. Spine, 1999; 24 (21): 2268.

11. Wong AP, Smith ZA, Lall RR, Bresnahan LE, Fessler RG: The microendoscopic decompression of lumbar stenosis: a review of the current literature and clinical results. Minimally Invasive Surgery, 2012; 2012: 325095.

12. Yaman O, Ozdemir N, Dagli AT, Acar E, Dalbayrak $\mathrm{S}$, Temiz C: A comparison of bilateral decompression via unilateral approach and classic laminectomy in patients with lumbar spinal stenosis: a retrospective clinical study. Turk Neurosurg. 2015; 25: 239-45.

13. Young $S$, Veerapen $R, O$ 'Laoire SA: Relief of lumbar canal stenosis using multilevel subarticular fenestrations as an alternative to wide laminectomy. Neurosurgery, 1988; 23: 628-633.

14. Zhang L, Miao HX, Wang $Y$, Chen AF, Zhang T, Liu $X G$ : Limited unilateral decompression and pedicle screw fixation with fusion for lumbar spinal stenosis with unilateral radiculopathy: a retrospective analysis of 25 cases. Journal of Korean Neurosurgical Society, 2015; 58 (1): 65.

\section{Additional Information}

Disclosures: Authors report no conflict of interest.

Ethical Review Board Approval: The study was conformed to the ethical review board requirements.

Human Subjects: Consent was obtained by all patients/participants in this study.

Conflicts of Interest:

In compliance with the ICMJE uniform disclosure form, all authors declare the following:

Financial Relationships: All authors have declared that they have no financial relationships at present or within the previous three years with any organizations that might have an interest in the submitted work.

Other Relationships: All authors have declared that there are no other relationships or activities that could appear to have influenced the submitted work. 


\section{AUTHORS CONTRIBUTIONS}

\begin{tabular}{|l|l|l|}
\hline Sr.\# & Author's Full Name & Intellectual Contribution to Paper in Terms of: \\
\hline 1. & Taimoor Anwar & Study design and methodology. \\
\hline 2. & Muhammad Abd-ur-Rehman & Paper writing, referencing, and data calculations. \\
\hline 3. & Urwa Tanveer Ahmad & Data collection and calculations. \\
\hline 4. & Anum Wahab & Analysis of data and interpretation of results etc. \\
\hline 5. & Ammar Anwer & Literature review and manuscript writing. \\
\hline 6. & Qura Tul Ain Tariq & Analysis of data and quality insurer. \\
\hline 7. & Zunera Tanveer & Literature review. \\
\hline 8. & Mohammad Zahid & Paper overall editing. \\
\hline
\end{tabular}

